\title{
Temporal Stability and Within-Subject Consistency of Nicotine Withdrawal Symptoms
}

\author{
James C. Tate, \\ Ovide F. Pomerleau, \\ and Cynthia S. Pomerleau
}

University of Michigan Behavioral Medicine Program

\begin{abstract}
This study tested the temporal stability and within-subject consistency of the Diagnostic and Statistical Manual-III-R (DSM-III-R) nicotine withdrawal symptoms in 39 (21 female, 18 male) regular smokers. Subjects provided withdrawal symptom ratings while smoking ad libitum and during two 48-hour abstinence periods that were separated by 1 week. All but two symptoms, increased eating and hunger, demonstrated adequate temporal stability (i.e., stability coefficients $\geq .70$ ). Withinsubject consistency was found for impatience, anxiety, difficulty concentrating, and irritability. The total (average) score emerged as the most stable and consistent indicator of withdrawal. Results of a cluster analysis suggest the presence of two groups of smokers based on the withdrawal experience. Possible reasons for variability in nicotine withdrawal symptom reports are discussed.
\end{abstract}

During the past decade the nature of the nicotine withdrawal syndrome has been the subject of some considerable scrutiny. Hughes, Hatsukami, and colleagues have been extremely influential in this area, and the DSM-III-R (American Psychiatric Association, 1987) diagnostic criteria for nicotine withdrawal are directly based on the findings of their research. One important aspect that has received scant attention is the consistency with which specific withdrawal symptoms appear during distinct periods of smoking abstinence. To our knowlcdge, only one study reported on the consistency of the nicotine withdrawal syndrome.

Hughes, Hatsukami, Pickens, and Svikis (1984) reported on the consistency of withdrawal symptoms using three subjects and an $\mathrm{ABAB}$ single-subject design. Ten measures of withdrawal were taken daily during two consecutive 48-hour ad libitum (A) and 96-hour abstinence (B) trials. A consistent change was scored if a symptom occurred during abstinence, returned to baseline, and recurred during the second trial for all three subjects. Six of 10 symptoms met this criterion: decreased heart rate, inscmnia, caloric intake, irritability, restlessness, and

Preparation of this manuscript was supported by National Institute on Drug Abuse Grant DA 06529 and by National Cancer Institute Grant CA 42730 to the second author.

Correspondence and requests for reprints should be sent to Chris C. Tate, Department of Psychology, P.O. Box 87, Middle Tennessee State University, Murfeesboro, TN 37132. 
drowsiness. Symptoms not demonstrating such consistency were orthostatic response, craving, and difficulty concentrating in one subject and anxiety in two subjects. In addition to these measures, two total withdrawal discomfort measures, the Total Mood Disturbance Scale of the Profile of Mood States (McNair, Lorr, \& Droppleman, 1971) and a composite score based on the Withdrawal Symptom Scale (Shiffman \& Jarvik, 1976), were utilized. Both of these measures showed consistent changes. Interestingly, only two of six affective or cognitive symptoms met the authors' criterion of consistent change (i.e., irritability and restlessness). Hughes et al. (1984) interpreted these results as demonstrating the temporal stability of the nicotine withdrawal syndrome and suggested that any within-subject inconsistency in withdrawal from one abstinence period to another is due to setting, expectancy, and method of cessation.

In a recent update of the nicotine withdrawal syndrome, Hughes and Hatsukami (1992) suggested that insomnia, craving, decreased heart rate, and hunger/weight gain, because of their prolonged time course, might not be valid nicotine withdrawal symptoms. Instead, they might be due to the offset of nicotine effects. Three of these "symptoms" were among the six demonstrating consistency in Hughes et al. (1984). This update suggested that consistency had been demonstrated only by irritability, restlessness, and drowsiness, and it challenged the authors' own assertion that the nicotine withdrawal syndrome possessed within-subject consistency.

The study presented here represents an investigation of the temporal stability and within-subject consistency of the DSM-III-R nicotine withdrawal symptoms using both measures and methodology different from that of Hughes et al. (1984). The major innovative features of this study include a revised version of the Hughes and Hatsukami Nicotine Withdrawal Forms (NWF; Hughes \& Hatsukami, 1986), a longer interval between abstinence periods, and a considerably larger sample size.

\section{METHOD}

\section{Subjects}

Subjects were 39 (21 female, 18 male) cigarette smokers who responded to a newspaper advertisement and who met stringent inclusion criteria: years smoked $\geq 3$, smoking rate $\geq 15$ cigarettes per day, Fagerstrom Tolerance Questionnaire (FTQ; Fagerstrom, 1978) score $\geq 5$, baseline expired alveolar carbon monoxide (CO) level $\geq 15 \mathrm{ppm}$, between the ages of 21 and 40 , within $20 \%$ of ideal body weight as assessed by measuring height and weight and consulting actuarial tables, no alcohol/drug abuse within the past 3 years as assessed via selfreport, and physically healthy as assessed by a physical examination, electrocardiogram, and standard laboratory tests. Subjects were screened initially over the telephone and informed that the study would involve two 48-hour abstinence periods. Few potential subjects declined to participate when informed of this requirement. 


\section{Measures}

A battery of baseline measures assessing smoking history, nicotine dependence (FTQ), smoking motives, depression (Center for Epidemiological Studies Depression, CES-D; Weissman, Sholomakis, Pottenger, Prushoff, \& Locke, 1977), and personality traits (State-Trait Anxiety Inventory-Trait: Speilberger, Gorsuch, \& Lushene, 1970; Tridimensional Personality Questionnaire (TPQ): Cloninger, Pryzbeck, \& Svrakic, 1991) was completed by all subjects. In addition, a revised version of the NWF, the Bipolar Nicotine Withdrawal Scale (BNWS) was used to assess nicotine withdrawal.

The BNWS includes several psychometric refinements designed to increase the validity of withdrawal symptom self-ratings. This instrument consists of 16 bipolar $100 \mathrm{~mm}$ visual analog scales (VAS) that were used to provide more continuous measures of withdrawal symptoms. All items are scored on a scale that ranges from -50 (least withdrawal) to +50 (most withdrawal), with the midpoint of each VAS being scored as 0 . Eleven of the items are reverse scored. A bipolar item format was chosen in order to avoid prompting subjects as to which symptoms to report. To guard against order and memory effects, the 16 items were divided into four blocks consisting of 4 items each, and the blocks were arranged to form four different orders of item presentation. Although the instrument consists of 16 items, only the 9 that tap DSM-III-R diagnostic criteria for nicotine withdrawal (i.e., craving, irritability, impatience, anxiety, restlessness, increased hunger and eating, decreased heart rate (HK), and difficulty concentrating) and total score (i.e., the mean of these 9 items) were included in the analyses. The remaining 7 items served as distractors to avoid prompting subjects. Irritability, anxiety, restlessness, increased eating, and hunger were reversed-scored items. Although the time frame for reporting withd rawal symptoms can vary, in this study subjects gave ratings for the past 24 hours at baseline and separate ratings for the first and second 24 hours of abstinence within each of two 48-hour abstinence periods. This choice of time frame reflects our interpretation of past research (e.g., Hughes \& Hatsukami, 1986; Hatsukami, Hughes, Pickens, \& Svikis, 1984), demonstrating that several acute withdrawal symptoms peak within the first 48 hours of nicotine abstinence. Preliminary psychometric analyses involving the BNWS total score demonstrated adequate internal consistency (i.e., $.70 \leq$ coefficient $\alpha \leq .79$ ).

\section{Procedure}

Subjects were seen on three separate occasions: at baseline, after 48 hours of abstinence, and 1 week later after an additional 48 hours of abstinence. At baseline, subjects completed several questionnaire measures, including the BNWS. Subjects smoked one usual brand cigarette 30 minutes prior to making withdrawal symptom ratings. Subsequently, subjects were contacted by telephone and instructed to stop smoking cold turkey at midnight and to remain abstinent for 48 hours prior to the second session. At that session, abstinence was verified via expired alveolar $\mathrm{CO}(\leq 10 \mathrm{ppm})$, and subjects provided retrospective ratings of withdrawal for each abstinence day using the BNWS. Subjects were 
Table 1. Subject Characteristics

\begin{tabular}{lccccrr}
\hline & \multicolumn{2}{c}{ Women } & \multicolumn{2}{c}{ Men } & \multicolumn{2}{c}{ Total } \\
Variable & $\boldsymbol{M}$ & $(\boldsymbol{S D})$ & $\boldsymbol{M}$ & $(\boldsymbol{S D})$ & $\boldsymbol{M}$ & $(\boldsymbol{S D})$ \\
\hline Age (Years) & 24.76 & $(5.21)$ & 25.94 & $(5.29)$ & 25.31 & $(5.21)$ \\
Smoking Rate (Cigarettes/Day) & 22.43 & $(3.79)$ & 24.94 & $(5.38)$ & 23.59 & $(4.71)$ \\
Baseline CO (ppm) & 29.10 & $(12.49)$ & 24.94 & $(9.30)$ & 27.18 & $(11.19)$ \\
FTQ & 6.10 & $(2.26)$ & 7.44 & $(1.50)$ & 6.72 & $(2.04)$ \\
$n$ & 21 & & 18 & & 39 & \\
\hline
\end{tabular}
naire.

Note. $\mathrm{CO}=$ carbon monoxide, $\mathrm{Ppm}=$ parts per million, FTQ $=$ Fagerstrom Tolerance Question-

instructed to begin smoking again and were observed smoking a cigarette at the end of the session to verify resumption of smoking. In exactly 1 week, the abstinence procedure was repeated.

\section{RESULTS}

\section{Preliminary Analyses}

Table 1 contains demographic and smoking-related variables for the total sample and separately for women and men. A multivariate analysis of variance (MANOVA) testing for gender differences on these variables was nonsignificant, $F(4,34)=1.66, p>.05$. Generally, the sample consisted of young, healthy smokers who reported being moderately dependent on nicotine. A MANOVA testing for sex differences in withdrawal symptom self-ratings was nonsignificant, $F(18,20)=.68, p>.05$. Consequently, data for men and women were combined.

\section{Major Analyses}

\section{Temporal Stability}

To assess temporal stability, the following procedure was followed. First, because it was predicted that averaged scores would be more stable than scores for either of the individual 24-hour time frames, first and second 24-hour ratings within each abstinence period were averaged. Second, baseline scores were subtracted from these averaged scores to yield difference scores for each 48-hour abstinence period. Third, difference scores for the first abstinence period were correlated with difference scores from the second abstinence period. Table 2 contains the resulting means and temporal stability coefficients for individual item and total BNWS scores. The temporal stability coefficients ranged from a low of .60 (increased hunger) to a high of .88 (total withdrawal score).

\section{Within-Subject Consistency}

To assess within-subject consistency, difference scores were derived as described before. Next, the frequency and percent of subjects reporting any in- 
Table 2. Means (Standard Deviations) and Temporal Stability Coefficients for Total Withdrawal and Individual Symptom Difference Scores

\begin{tabular}{lrrrrr}
\hline Measure & $\boldsymbol{M}_{\mathbf{1}}$ & $(\boldsymbol{S D})$ & $\boldsymbol{M}_{\mathbf{2}}$ & $(\boldsymbol{S D})$ & $\boldsymbol{r}^{*}$ \\
\hline Total & 15.05 & $(12.50)$ & 13.23 & $(13.00)$ & .88 \\
Irritability & 24.87 & $(26.79)$ & 20.29 & $(24.52)$ & .87 \\
Decreased HR & 2.01 & $(11.03)$ & -0.44 & $(11.22)$ & .84 \\
Anxiety & 22.09 & $(27.61)$ & 20.60 & $(25.80)$ & .85 \\
Craving & 10.21 & $(21.62)$ & 6.58 & $(19.07)$ & .85 \\
Difficulty Concentrating & 22.03 & $(25.16)$ & 18.88 & $(21.92)$ & .84 \\
Impatience & 22.90 & $(25.21)$ & 22.10 & $(26.54)$ & .78 \\
Restlessness & 8.29 & $(20.83)$ & 6.03 & $(21.00)$ & .73 \\
Increased Eating & 14.72 & $(16.67)$ & 13.96 & $(17.62)$ & .62 \\
Hunger & 8.31 & $(17.58)$ & 11.05 & $(20.33)$ & .60 \\
\hline
\end{tabular}

Note. $\mathrm{HR}=$ heart rate.

$*$ All $p$ s $=.0001$.

crease during both abstinence periods (concordant increases), the frequency and percent of subjects reporting any decrease during both abstinence periods (concordant decreases), and the frequency and percent of discordant cases were determined for each withdrawal symptom and the total score. These calculations were followed by $\chi^{2}$ goodness-of-fit tests to determine whether significantly more subjects reported concordant increases in withdrawal severity than combined concordant decreases and discordant experiences. Table 3 contains the results of these calculations. The total withdrawal discomfort score demonstrated the best consistency, with $92 \%$ of subjects reporting concordant increases in severity. For four of the nine individual withdrawal symptoms and the total score, the $\chi^{2}$ results were significant, indicating significantly more subjects reported concordant increases in withdrawal severity on these variables. Percent-

Table 3. Estimates of Within-Subject Consistency for Total Withdrawal and Individual Symptom Difference Scores

\begin{tabular}{lcccc}
\hline Symptom & $\begin{array}{c}\text { \% Concordant } \\
\text { Increases }\end{array}$ & $\begin{array}{c}\text { \% Concordant } \\
\text { Decreases }\end{array}$ & $\begin{array}{c}\text { \% Discordant } \\
\text { Cases }\end{array}$ & $\begin{array}{c}\boldsymbol{X}^{2} \\
(\boldsymbol{d} f=\mathbf{f}=\mathbf{n}=\mathbf{3 9})\end{array}$ \\
\hline Total & 92 & 5 & 3 & $24.64^{* * *}$ \\
Impatience & 77 & 13 & 10 & $11.30^{* * *}$ \\
Anxiety & 74 & 13 & 13 & $9.26^{* * *}$ \\
Difficulty Concentrating & 72 & 15 & 13 & $7.41^{* *}$ \\
Irritability & 69 & 13 & 18 & $5.77^{*}$ \\
Increased Eating & 64 & 8 & 28 & 3.10 \\
Craving & 62 & 21 & 18 & 2.08 \\
Hunger & 51 & 15 & 34 & .03 \\
Restlessness & 51 & 23 & 26 & .03 \\
Decreased HR & 36 & 28 & 36 & .47 \\
\hline
\end{tabular}

Note. HR $=$ heart rate.

${ }^{*} p<.05 . \quad{ }^{* *} p<.01 . \quad{ }^{* * *} p<.005$. 
ages of subjects reporting concordant increases in severity for eating, craving, hunger, restlessness, and decreased HR did not differ from what would be expected by chance. Interestingly, substantial percentages of subjects reported concordant decreases in symptomatology or discordant withdrawal experiences.

\section{Cluster Analysis}

Because the results presented here suggest that smokers may vary in terms of their withdrawal experiences, cluster analytic procedures were used in a preliminary effort to discover subtypes of smokers based on withdrawal symptomatology. Only data from the first 48 -hour abstinence period were used. This choice reflects the belicf that this abstinence period was more representative of abstinence in general because it was not contaminated by any recent quitting attempt. Using the Statistical Analysis System (SAS) statistical package, a cluster analysis (Ward's method; Ward, 1963) was performed using squared Euclidean distance as the similarity measure. Determination of the number of clusters was arrived at through inspection of the resulting dendrogram. The withdrawal symptom difference scores served as the clustering variables.

A clear two-cluster solution emerged (see Figure 1): a group numbering 32

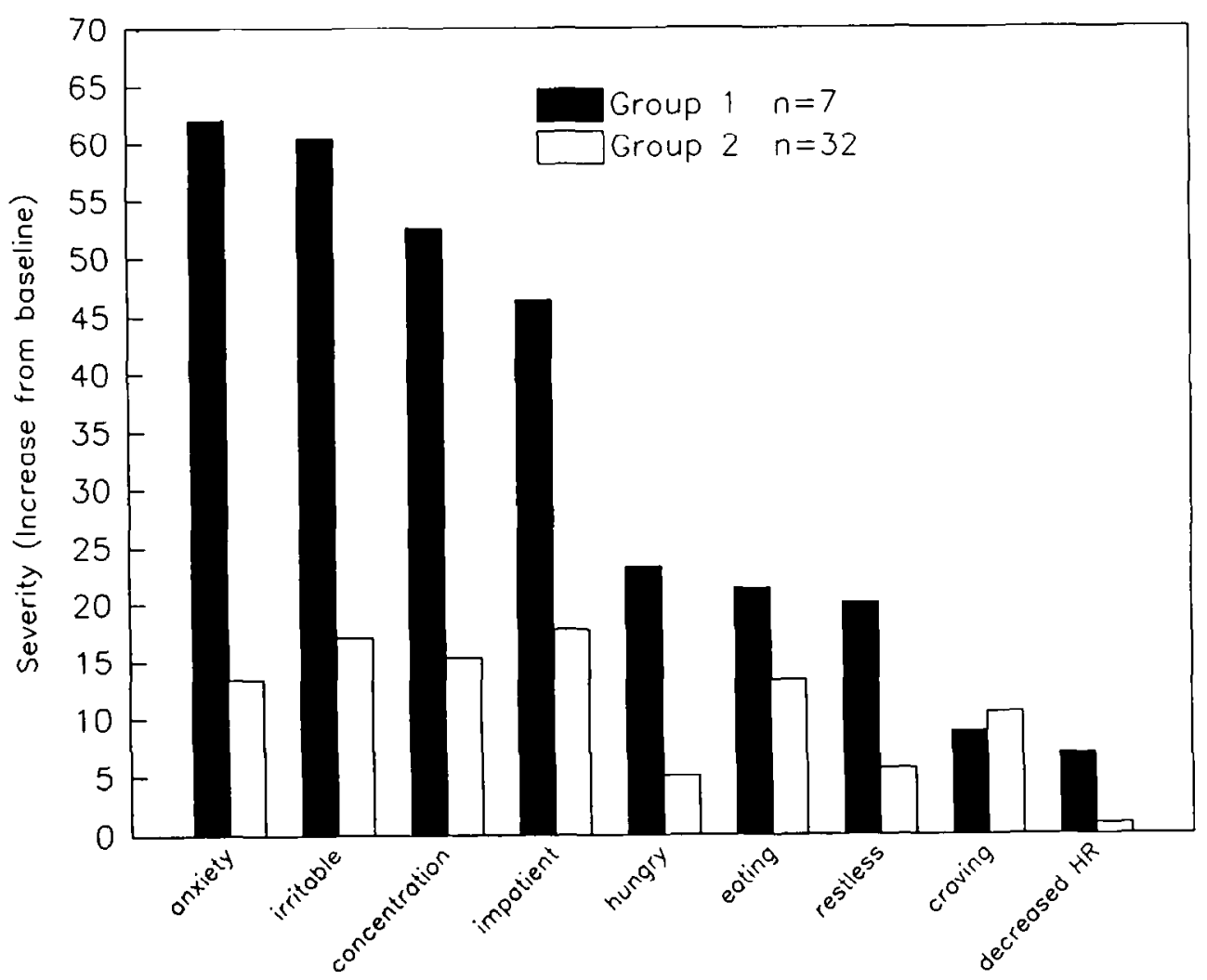

Figure 1. Profiles of Groups formed by Cluster Analysis. HR = heart rate. 
subjects ( 14 women, 18 men) and a smaller group numbering 7 subjects (3 women, 4 men). Univariate analyses of variance (ANOVAs) were used to determine on which withdrawal symptoms the two groups differed (i.e., to determine the basis of the cluster analytic solution). The Bonferroni procedure was used to adjust alpha for testwise Type I error (critical $\alpha=.0055$ ). The two groups differed on the following four symptoms: irritability, $F(1,37)=24.22, p=.0001$; impatience, $F(1,37)=8.93, p=.005$; difficulty concentrating, $F(1,37)=18.43$, $p=.0001 ;$ and anxiety, $F(1,37)=32.91, p=.0001$. In each case, the smaller group's mean withdrawal score was significantly larger.

Although the stringent inclusion criteria produced a homogeneous sample with respect to age and smoking history, three MANOVAs were performed to determine whether the two groups differed on selected baseline measures. Significant differences would help to further characterize the two groups. The first MANOVA, which tested for differences in age, baseline $\mathrm{CO}$, baseline smoking rate, and FTQ scores, was nonsignificant, $F(1,34)=.48, p=.75$, as was the second MANOVA, which tested for baseline differences in depression and trait anxiety scores, $F(2,36)=1.28, p=.29$, and the third MANOVA, which tested for differences on the various TPQ subscales, $F(15,23)=.43, p=.95$.

\section{DISCUSSION}

In the study presented here, the temporal stability and within-subject consistency of the DSM-III-R nicotine withdrawal symptoms were tested. Irritability, decreased heart rate, anxiety, craving, difficulty concentrating, impatience, and restlessness emerged as the most stable individual withdrawal symptoms. Increases in impatience, anxiety, irritability, and difficulty concentrating demonstrated the highest within-subject consistency. Anxiety, difficulty concentrating, irritability and impatience emerged as both highly stable and consistent. Overall, the total withdrawal score was found to be the single most stable and consistent index of withdrawal. These results are impressive given that each withdrawal symptom was measured by a single-item scale, a condition that typically limits the reliability of a measure.

Nicotine withdrawal is generally stable and consistent, but its nature and severity can differ from one smoker to another and from one abstinence period to another for some smokers and for some symptoms. Specifically, several subjects reported concordant decreases in withdrawal symptomatology or were discordant in their reports of withdrawal. This is perhaps the most intriguing finding of this study. For example, $21 \%$ of the subjects reported less craving during both abstinence periods compared to baseline, and $18 \%$ of the subjects reported greater than baseline craving during one abstinence period and less than baseline craving during the other.

Although the total withdrawal scores and some individual symptom scores demonstrated excellent stability and consistency, other symptoms did not. Moreover, for all symptons measured in this study, there was evidence of instability and inconsistency in some subjects. Some of this inconsistency may be due to measurement error, but other factors may be operative.

Two explanatory possibilities exist. First, the withdrawal experience may dif- 
fer from one abstinence period to another because of changes in setting, expectancies, and method of quitting, as Hughes et al. (1984) suggest. For example, those subjects reporting withdrawal symptom decreases during abstinence may have been more effective at avoiding situations associated with drug availability and smoking cues. Also, in the case of discordant withdrawal experiences, because subjects were not provided with a standard procedure for refraining from smoking during the two abstinence periods, the extent to which differences in quitting method could have influenced withdrawal symptoms is unknown. If such is the case, future research might most profitably target such variables as instructional sets and expectancies (Gottlieb, Killen, Marlatt, \& Taylor, 1987; Hughes, Pickens, Spring, \& Keenan, 1985) and cue reactivity (Niaura, Abrams, Demuth, \& Pinto, 1989) in an effort to understand nicotine withdrawal better and to develop better methods of relieving withdrawal.

Second, there may be subtypes of smokers who differ in the experience of withdrawal. That is, independent of setting, expectancies, method of cessation, and degree of dependence, smokers may differ with respect to the specific constellation of symptoms manifested during smoking abstinence. The clusteranalytic results presented earlier, although preliminary, clearly demonstrated that abstaining smokers could be differentiated based on their withdrawal experiences, independent of such factors as age, gender, nicotine dependence, mood state, and selected personality characteristics. (The selection of the sample for homogeneity on certain demographic variables, however, does temper this assertion somewhat). Specifically, the smaller subgroup of subjects (identified by cluster analysis) reported higher levels of irritability, impatience, difficulty concentrating, and anxiety along with comparable levels of craving, restlessness, hunger, eating, and decreases in heart rate, compared to the larger subgroup. The former set of four "marker" symptoms (i.e., irritability, impatience, difficulty concentrating, and anxiety) were the ones found to be most stable and consistent in the main study. Among the treatment implications of the cluster analysis findings is that patients may require augmentation of therapy to deal with affective disturbances that are independent of nicotine dependence. Clearly, this possibility warrants further attention.

Finally, some caveats with respect to the generalizability of our findings should be mentioned. First, temporary withdrawal from nicotine may be psychologically very different from withdrawal that is part of a cessation attempt. Thus, the extent to which the present results generalize to smoking cessation clinic patients is unknown. Second, the use of longer periods of abstinence separated by a longer period of resumption of smoking could result in very different findings. Future research is needed to determine how representative the present findings are of nicotine withdrawal in other settings.

\section{REFERENCES}

American Psychiatric Association. (1987). Diagnostic and statistical manual of mental disorders (3rd. ed., rev.). Washington, DC: Author.

Cloninger, C.R., Pryzbeck, T.R., \& Svrakic, D.M. (1991). The tridimensional personality questionnaire: U.S. normative data. Psychological Reports, 69, 1047-1057. 
Gottlieb, A.M., Killen, J.D., Marlatt, G.A., \& Taylor, C.B. (1987). Psychological and pharmacological influences in cigarette smoking withdrawal: Effects of nicotine gum and expectancy on smoking withdrawal symptoms and relapse. Journal of Consulting and Clinical Psychology, 55, 606608.

Fagerstrom, K.O. (1978). Measuring degree of physical dependence to tobacco smoking with reference to individualization of treatment. Addictive Behaviors, 3, 235-241.

Hatsukami, D.K., Hughes, J.R., Pickens, R.W., \& Svikis, D. (1984). Tobacco withdrawal symptoms: An experimental analysis. Psychopharmacology, 84, 231-236.

Hughes, J.R., \& Hatsukami, D.K. (1986). Signs and symptoms of tobacco withdrawal. Archives of General Psychiatry, 43, 289-294.

Hughes, J.R., \& Hatsukami, D.K. (1992). The nicotine withdrawal syndrome: A brief update. International Journal of Smoking Cessation, 1, 21-26.

Hughes, J.R., Hatsukami, D.K., Pickens, R.W., \& Svikis, D. (1984). Consistency of the tobacco withdrawal syndrome. Addictive Behaviors, 9, 409-412.

Hughes, J.R., Pickens, R.W., Spring, W., \& Keenan, R.M. (1985). Instructions control whether nicotine will serve as a reinforcer. The Journal of Pharmacology and Experimental Therapeutics, 235, $106-112$.

McNair, A.D., Lorr, M., \& Droppleman, L.F. (1971). Manual for the profile of mood states. San Diego: Educational and Industrial Testing Service.

Niaura, R., Abrams, D., Demuth, B., \& Pinto, R. (1989). Responses to smoking-related stimuli and early relapse to smoking. Addictive Behaviors, 14, 419-428.

Shiffman, S., \& Jarvik, M.E. (1976). Smoking withdrawal symptoms in two weeks of abstinence. Psychopharmacology, 50, 35-39.

Spielberger, C.D., Gorsuch, R.L., \& Lushene, R.E. (1970). Manual for the state trait anxiety inventory. Palo Alto, CA: Counseling Psychologists Press.

Ward, J. (1963). Hierarchical grouping to optimize an objective function. Journal of the American Statistical Association, 58, 236-244.

Weissman, M.M., Sholomakis, D., Pottenger, M., Prushoff, B.A., \& Locke, B.Z. (1977). Assessing depressive symptoms in five psychiatric populations: A validation study. American Journal of Epidemiology, 106, 203-214. 